\title{
Is There Such a Thing as Orthogonyms?
}

\author{
Wes Raykowski \\ Griffith University, Australia \\ wes.rajkowski@alumni.griffithuni.edu.au
}

\begin{abstract}
The article explores the cognitive semantics thesis that lexical expressions function as points of access to vast repositories of schematic concepts arising from embodied experience. By comparing various forms of communication, I find that expressions in art, science, and technology display a pattern in which expressions prompting the sense of intensity, magnitude and force are often combined into products with expressions that prompt the sense of extent, multitude and displacement. This pattern seems to be largely absent from natural language. I argue that lexical items activate the same pattern, though in a less direct way.
\end{abstract}

\section{Keywords}

orthogonyms - intensity - extent - cognitive products

Most of our evidence has come from language - from the meanings of words and phrases and from the way humans make sense of their experiences.

(Lakoff and Johnson 2003: 115) 
There is a curious pattern involving sensations - they are experienced only when their magnitude is sufficiently high and their extent is sufficiently large. If one of those factors is missing, there can be no sensation. Thus, sound can be experienced only if it has an adequate volume and a sufficiently long duration, and touch can be experienced only if the pressure exceeds a certain threshold and the surface is sufficiently large. The same pattern of magnitude paired with multitude and cause paired with effect can be found in engineering, economics, and education. ${ }^{1}$ However, nowhere is the presence of this pattern more obvious than in the sciences.

It is therefore surprising that this pattern, which is so common in scientific and technical literature, seems to be absent from natural language. In light of the 'generalization commitment' and 'cognitive commitment' (Evans and Green 2006: 27-50) on which the entire cognitive linguistics enterprise rests, this absence sounds implausible. The solution to this problem could be the hypothesis of orthogonyms based on the generalization of sensory maps proposed in this paper. This hypothesis assumes that various somatosensory maps in the brain are active representations of spatial relations between receptors distributed around various surfaces of the body. These spatial relations are coded in terms of the activation of neurons (intensity), the number of activated neurons (extent) and the spatial arrangement of activated neurons (pattern). The principal claim is that various properties of those maps define not

1 In thermodynamics and engineering, the pattern is confined to dyads such as intensiveextensive properties, effort-flow variables, force-displacement and across-through conjugates. In economics, the same pattern appears under names such as intensive and extensive production systems, margins, and growth as well as the value and volume of products and the cost and value of sales. Identical patterns can be found in education in the way people talk about the magnitude and multitude of quantities and dependent and independent variables. Note that every such pair represents two distinct ways of thinking. The first term denotes causes (e.g., force, tension or effort of some magnitude), while the second term draws attention to the effects (e.g., displacement, extension, or flow of some extent). What is curious is that the members of those dyads are neither opposed to nor aligned with each other but are in an orthogonal relationship (refer to Section 5). When associated together (multiplied), those two patterns give rise to products (Section 5). For examples in thermodynamics, refer to "Force as a flow variable" by Fairlie-Clarke (1999) and "Analogy theory for a system approach to physical and technical systems" by Hezemans \& Geffen (1991); for an example in economics, refer to "A practical guide to trade policy analysis" by Bacchetta et al. (2012); and for an example in education, see "Understanding whole numbers" by Nunes and Bryant (2009). 
only the character of sensations but also all subsequent associations including diverse forms of expression with natural language among them.

The most important property of these maps is the orthogonal relationship between intensity and extent, which represent two contrasting yet complementary concepts. They can be visualized with brackets as $((((\ldots))))$ for intensity and ()()()() ... for extent. The arrangement of brackets suggests that intensity is experienced as a nesting within a unit and extent as the repetition of a unit. Note that the intensity and extent (hence nesting and repetition) are not antonyms as they are not in opposition to each other. They remain in the orthogonal relationship, which is evident in their expressions in natural language; intensity typically refers to the vertical and extent to the horizontal axes (see Section 5 for elaboration). For this reason they are referred to as orthogonyms.

The concepts of intensity and extent serve as prototypes for all expressions displaying nested and repetitive structures. In the context of simple adjective phrases, the orthogonyms can be described as aspects of a property (attributed to the object/subject) displaying the orthogonal characteristics encapsulated by the notion of nesting and repetition. Pairs such as the intensity of a color and its area; the pitch of a note and its duration; the intensity of pain and its extent; the sweetness of a food and its volume; the power of feeling and its duration; the potency of a medication and the number of pills; the violence of protesters and the crowed size; the gravity of a crime and the number of offences; or feelings of animosity and their duration - are all examples of orthogonyms. They all come in pairs of 'intensive-extensive' orthogonyms defined in the context of a property.

If the experiences of most if not all sensory modalities involve patterns of some intensity and extent, then identical patterns must be recreated while simulating (imagining, recalling, remembering) those experiences (see Section 6). This in turn means that both aspects must be specified in the expressions (e.g., sentences, paintings, music pieces, mathematical equations, etc.) used to initiate such simulations. The way in which this is coded is likely to vary between different modes of communication. In artistic, scientific and technical expressions, orthogonyms are clearly defined and always present (see Section 7.1 and 7.2). This is not the case, at least not always, in natural language, in which orthogonyms are often suggested indirectly (Section 7.3). This makes their presence less obvious. There is, therefore, a temptation to view intensity and extent, hence orthogonyms and their products, from the perspective of science and mathematics - instead of seeing them as the result of cognitive processes preceding the emergence of communication.

The sensory beginnings of communication processes and their emergent nature can potentially explain the workings of natural language - the function 
of intensity and extent in adjectives and adverbs; the role they play in synonyms, antonyms and opposition in general; and their effect on the structure of phrases, clauses and sentences. Most importantly, the notion of orthogonyms helps to unify various fields of research and explain the origin of common concepts used in those disciplines. One example is the notion of products in natural language and mathematics, which are likely to be derived from the cognitive interpretation of products. Cognitive products reflect the idea that both intensity and extent are required for sensory experiences to take place. The product is, therefore, defined as a specific association of orthogonyms representing a particular property or aptitude of an object or subject (Section 5 ). It is important to note that the hypothesis offered in this article is in many respects compatible with the Cognitive Grammar schemas developed by Langacker (1987). In closing, the caveat is in order. Like all emergent theories, the proposed hypothesis can illuminate only the foundational issues of language and its organization. The extraordinary variation of linguistic expressions can be explained by the top-down analysis of language.

Owing to the multidisciplinary character of the argument and its ambitious scope, this article had to be drastically reduced in favor of more in depth discussion. As a result, some issues listed in the preceding paragraph are not discussed and the linguistic investigation of orthogonyms is limited to a few rudimentary noun phrases in English. The important issue of sensory patterns is excluded and the philosophical justification for the argument is omitted from the article. For the same reason, the use of orthogonyms in science and technology is not discussed to any significant extent and the discussion of simulation is limited to only steady states. To improve the flow of the argument, the peripheral topics (e.g., orthogonyms in thermodynamics and engineering) are located in the footnotes. One final comment - the objective of the article is to introduce various cognitive concepts defined in the context of somatosensory maps in the brain. This resulted in a significant part of the article being devoted to aspects of neuroscience. To make the article more relevant to linguistics, the discussion of those topics is restricted to conceptual implications of brain organization.

The article is based on the book "Conceptual Understructure of Human Experience" (Raykowski 2014) and the paper titled "Layers and Levels: What a Column of Water Tells Us about Human Cognition" (Raykowski 2018).

In the course of day-to-day studies of linguistics, it is easy to forget that natural language is only one of many ways in which human individuals can express 
their private sensory experiences. Other forms include science and arts as well as gestures, posture, and facial expressions. Some media have a more obvious structure than natural language. Consider the act of painting a green apple as an example of visual expressions. Whenever colors are applied to a canvas, two important aspects are always fully present: the intensity of a color and its area. Note that it is not possible to express or see colors that have no intensity; such colors would appear to the eye as blackness. It is also impossible to express and see colors that have no area. Therefore, when an object (e.g., a green apple) is painted, both aspects of color are always expressed simultaneously.

Now, compare the painting of a green apple with the linguistic phrase green apple. Neither the extent nor the intensity of the color is mentioned in the phrase. This cannot be right, as both aspects of human experience must be conveyed irrespective of the mode of expression. ${ }^{2}$ One likely explanation is that the phrase green apple is an abbreviation ${ }^{3}$ of a more complete phrase that specifies not only the properties of the apple but also their changes. Such an expression would read something like the average and unchanging intensity of the apple's green surface. If not mentioned, the intensity of a color is assumed to be average, typical or just salient, and the extent of the color is the apple's surface - both of which do not change with time. This interpretation applies to all properties of the apple. If those properties are other than average, their description must be appropriately modified from, for example, crisp, sweet and small to quite crisp, very sweet and rather small. The crispness of the apple is described as satisfactory (quite crisp), and the degree of its sweetness is defined with the intensifier very. The extent in both cases is given not by the apple's surface but by its volume. Size, on the other hand, is a combination of the volume and diameter of the apple (e.g., it fits in a grasping hand and/or an opening mouth), and its magnitude is modified with the word rather. Note that if there is more than one apple, the extent of each property is multiplied by the number of apples, but their respective intensities remain unchanged. Additionally, observe that the phrase green apple does not indicate the rate of property change, which leads to the assumption that both intensity and its

2 As previously mentioned, the claim has its origins in the cognitive commitment. Evans and Green outline the issues as follows: “[...] it follows from the 'Cognitive Commitment' that language and linguistic organisation should reflect general cognitive principles rather than cognitive principles that are specific to language" (2006: 41).

3 Tyler and Evans describe the matter as follows: "Language (lexical items and the syntactic arrangements in which they occur) radically underdetermines the rich interpretations regularly assigned to naturally occurring utterances. A consequence of this is the assumption that lexical entries, albeit crucial, act merely as prompts for meaning construction, and that meaning construction is largely a conceptual process, involving elaboration and integration of linguistic and non-linguistic information in a highly creative way [...]" (2003: 3). 
extent do not vary over time. As in the case of intensity and extent, the rate of change is rarely expressed.

Upon hearing such highly abbreviated sentences, most readers tend to recreate (in the sense of covert mental simulations) more vivid scenery by recalling their own sensory experiences of handling apples. With it, they revive many aspects of the experience that are missing from underspecified linguistic expressions. The processes of recalling, remembering and imagining are interpreted in cognitive linguistics as embodied simulations. According to simulation theories, recreating sensory experiences involves partial activation of the resources with which those sensations were initially experienced (Barsalou 1999). An important part of those resources are somatosensory maps in the brain. A discussion of brain structure is therefore essential for the understanding of orthogonyms and their role in the simulation processes. These issues are considered in the next four sections starting with the importance of topographic/topological maps for sensory organization.

\section{Organization of Sensory Experience}

The importance of sensations for cognition has been grossly underestimated by research communities. Sensations have been typically described as unstructured, unorganized, primitive and raw input (signals or data) for perception and subsequent cognitive processes. It is also widely assumed that sensations do not convey any meaning. These notions suggest that sensations are unorganized and passive, which, I argue, is not accurate. There is rich literature on somatosensory and motor maps. The research confirms that the topological (and topographic) organization of sensations is fundamental to brain operation (e.g., Penfield \& Boldrey 1937; Grodd et al. 2001; Bednar and Wilson 2015).

In contrast, contemporary literature typically interprets the brain as a neural network whose organization is governed by the economy principle (Wang and Clandinin 2016: R1101) first expressed by Ramón (1899). The proponents of this interpretation pay little attention to the low signal transmission rate along neurons. In the case of sensory and motor neurons of substantial lengths, the issue of transmission cannot be ignored. The distance between the body's periphery and the brain can reach two meters in tall humans, five meters or more in giraffes and up to 30 meters in whales (Smith 2009). Such distances are significant if one considers the speed of signal propagation along neurons, which is extremely slow compared to the transmission along wire networks. This is likely to have many consequences for the organization of neurons in the brain and for the processing sensations and activation of motor faculties (Smith 2000: 29-34). 
Raykowski (2014) argues that cortical maps are an organizational necessity for all systems involving slow-transmission circuits, which in the case of neurons vary from less than a meter up to 120 meters per second (Caldwell 2009). Neuronal circuits are therefore highly dependent on the length of their connections. According to Raykowski, sensory maps in the brain (together with other arrangements) guarantee that all elements of spatially related stimuli (e.g., all parts of a face, hand or any other extremity) are activated and processed almost simultaneously. This can be ensured only if the length of the neurons constituting those maps is identical or at least similar, which is the most critical feature that differentiates neural maps from networks. ${ }^{4}$

Other consequences of the length disparity include slowing down the reaction time of organisms and, if the disparity is too excessive, a situation in which sensory patterns are perceived as unrelated. One could say that the neuron slow-transmission rate necessitates the topological organization of cortical maps. The maps are also essential for the intentional (as opposed to reflexive) movement of the body. To scratch the knee, for example, the brain has to recreate all spatial relations between various parts of the knee, trunk, arm, hand, and fingers. Topological and topographic maps are therefore the most fundamental meaning-creating feature of the brain. ${ }^{5}$ The circuits connecting various maps can then be seen as a means of associating spatial relations coded in the individual maps of different modalities. To reduce the body's reaction time, all circuits should also be as short and fast as possible.

The cognitive consequences of topological and topographic organization are discussed in the next section starting with a short analysis of geographic maps and their arrangement and properties with the emphasis on their spatial characters. The discussion is critical for the understanding of orthogonyms, the concept of which is defined in the context of sensory maps.

\section{Role of Sensory Maps in Cognition}

Sensory maps are important for movement. To move consciously, many complex organisms need to be aware of spatial relations not only outside but also

4 The tendency to interpret the brain as a network has been reinforced by computer models. Note that most computer networks are not sensitive to the length of their connections. In this context, neural maps can be defined as modal networks in which the connections between sensors and their respective maps are of the same or similar length. Only under this condition the nodes of the network can be arranged topographically or topologically. The neural maps are therefore a special case of the neural networks.

5 The presence of slow- and fast-adapting neurons do not affect the argument. 
inside their own bodies. The knowledge of spatial relations, I argue, is provided by topographic and topological somatosensory maps that are understood here as spatially organized collections of sensory cells (and their proxies) grouping neurons of identical sensory endings known as receptors. The maps reflect the spatial distribution of sensory receptors throughout the body. In this context, space and spatial relations are experienced as patterns of cells that are excited to contrasting degrees. To illustrate these issues, I first discuss the organization of cartographic maps.

If symbols are ignored, geographical maps are nothing more than collections of spatial elements (e.g., dots in printed images or pixels on the screen). The map in Figure 1 is a collection of identical hexagonal elements, all of which are highlighted (represented with white) or remain dark. With this simple twostate arrangement, the map can already be used to express a great variety of patterns and relations between them. The reader can easily recognize continents depicted in the figure. The information provided by such two-state representations depends on the number of elements constituting the map and it can be improved further by using an extended range of grey. Observe that the extent of patterns and their relations are expressed in the map entirely in terms of spatial elements.

I claim that there are many similarities between the map in Figure 1 and various sensory maps in the brain. Unlike geographic maps, patterns and their relations are coded in sensory maps by means of neuron activation. The basic

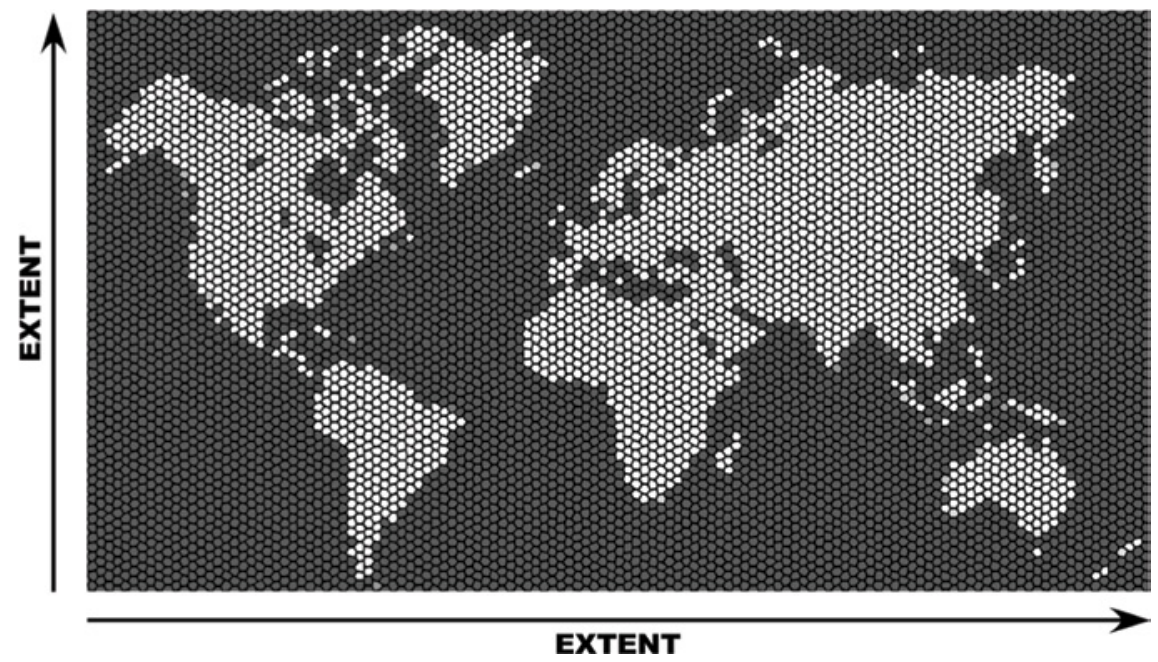

FIGURE 1 An example of a spatially organized map. The map is a collection of identical hexagonal elements; some elements are activated (represented by white), while others remain deactivated. 


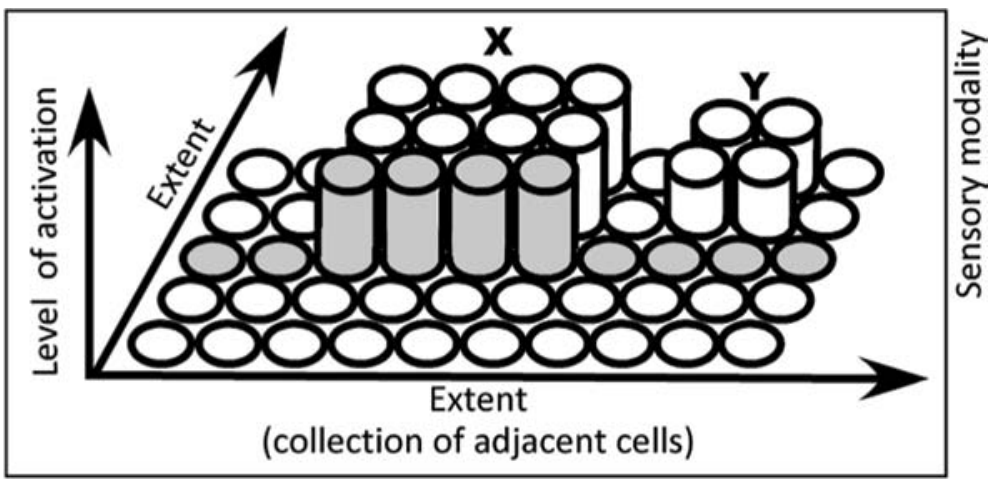

FIGURE 2 An example of a simplified sensory map with twelve cells activated to level X, four cells to level Y and the remaining inactive.

structure of sensory maps is depicted in Figure 2 showing a small collection of sensory cells. ${ }^{6}$ To indicate their highly interconnected nature, the cellular elements are shown touching each other. The contrasts are created by varying levels of cellular activation, which is depicted as cylinders whose height is proportional to excitation (e.g., the excitation of cells marked with $\mathrm{X}$ is greater than that of Y cells). The elements of the same or sufficiently similar activation represent a spatial pattern of a certain intensity (the height in the example) and a certain extent (plurality of activated cells). Note the vertical orientation and nested character of the activation (high excitation includes lower levels of excitation, which includes no excitation) and the horizontal and repetitive nature of cells distributed along the horizontal surface. The diagram suggests an orthogonal (perpendicular) relationship between the collection of cells and their activation. Note that the activation of elements in the map can be used to represent not only various spatial patterns (e.g., figures) but also changes from one pattern to another (e.g., moving figures or changing forms).

The peculiarities of sensory maps (such as their extent and intensity) and their relationship to the concept of orthogonyms are outlined in the next two sections.

\subsection{Extent of Sensation}

Perhaps the most characteristic aspect of all maps is their spatial character. The maps are collections of spatial elements (dots, pixels, cells) located on a surface. If they are arranged topologically or topographically, the maps can

6 To emphasize the issues at hand, various aspects of cortical maps (e.g., their layered structure) are ignored. 
represent spatial relations of the surfaces they reflect. The notion of space is therefore integral to all topological maps made of space-occupying elements. In the context of sensory maps, space can be understood as a collection of concatenated (in the sense of strong interconnections) nerve cells. Such space is tangible (biological cells), continuous (there is a negligible gap between all concatenated cells), homogeneous (all cells within a given modality are identical) and always present even when none of the cells is activated. The inactive cells give rise to the sense of emptiness, which stands for the absence of 'objects' that are able to cause sensory activation.

Collections of activated cells from which the presence of 'objects' is inferred indicate the size (extent) of those 'objects'. Cells with no (or lower) activation are perceived as the empty space between the 'objects'. Patterns of activation caused by the 'objects' divide the map into inner space, boundaries, and the common (public) space outside of them. The space inside and outside of those patterns is made of identical cells, hence the universal sense of space within any given modality. The varying intensity of cells creates contrasts not only between activated and deactivated cells but also between cells activated to different levels (e.g., see patterns $\mathrm{X}$ and $\mathrm{Y}$ in Figure 2). Activated cells are more salient than their counterparts with less or no activation. They give rise to the figure-background experience. There can be more than one figureground relation because the figures can have more than one background. Together, the intensity and extent of experience are interpreted as a property of the 'object'. The cells separating 'objects' depict spatial relationships with other 'objects' within the map.

In this view, 'objects' are associations of patterns of various sensory modalities routinely experienced together. As each sensory modality has its own maps, there are as many senses of space as there are sensory modalities. For example, the sense of space in vision (topographic space) is different from the sense of space in touch (topological space), which is different from smell and taste. The combination of various senses leads to incredibly rich and varied experience of space, 'objects', spatial relations and their changes (Raykowski 2014). Because 'objects' and the space around them are expressed with identical elements, the relations between objects (but also relations of relations), which are typically considered abstract, are as concrete as the 'objects' themselves.

The assemblies of concatenated elements (e.g., cells of cortical maps) impose a sense of plurality (multitude as opposed to magnitude) of identical elements, which can be added, subtracted, divided from or combined with other similar elements. This sense represents one aspect of orthogonal concepts the extent. The extent alone is of no consequence for cognition. No collection of identical elements can convey meaning unless those elements can be 
differentiated in some way. In the case of cortical maps, this is achieved by selectively varying levels of cell activation (excitation), which is the subject of the next section.

\subsection{Intensity of Sensation}

The notion of orthogonyms in this article is defined in the context of sensory maps, the hypothesis of which relies heavily on the concept of sensory intensity. Sensory representations require that the neuron cells constituting various sensory maps record and represent the intensity of the stimulus in a reliable and consistent manner. According to Gardner and Johnson (2012: 453):

The firing rates of sensory nerves encode the stimulus magnitude. The data in the two plots suggest that the neural coding of stimulus intensity is faithfully transmitted from peripheral receptors to cortical centers that mediate conscious sensation. (...) The number of action potentials per second recorded from a touch receptor in the hand is proportional to the amplitude of skin indentation. Each dot represents the response of the receptor to pressure applied by a small probe. The relationship between the neural firing rate and the pressure stimulus is linear. This receptor does not respond to stimuli weaker than $200 \mu \mathrm{m}$, its touch threshold. (...) Estimates made by human subjects of the magnitude of sensation produced by pressure on the hand increase linearly as a function of skin indentation. The relation between a subject's estimate of the intensity of the stimulus and its physical strength resembles the relation between the discharge frequency of the sensory neuron and the stimulus amplitude.

The article by Gardner and Johnson, ${ }^{7}$ together with papers and books on the subject of psychophysical measurement (e.g., Harnad 1987), reaffirms the common observation that humans can sense not only the extent (e.g., area or duration) but also the intensity of sensory experiences.

The concept of intensity is difficult to comprehend for a number of reasons. The experience of intensity is unlike that of extent. It represents a nested relationship. ${ }^{8}$ Nested arrangements are difficult to express, visualize and argue

7 See also Smith (2000).

8 Intensity is an example of the schema Mark Johnson refers to as SCALE: "Scales have a cumulative character of a special sort. If you are collecting money and have accumulated $\$ 15$, then you also have $\$ 10$ " (1987: 122). Raykowski $(2014,2018)$ refers to this arrangement as a NESTED schema because it applies to all nested concepts such as value, importance, magnitude, and level. 


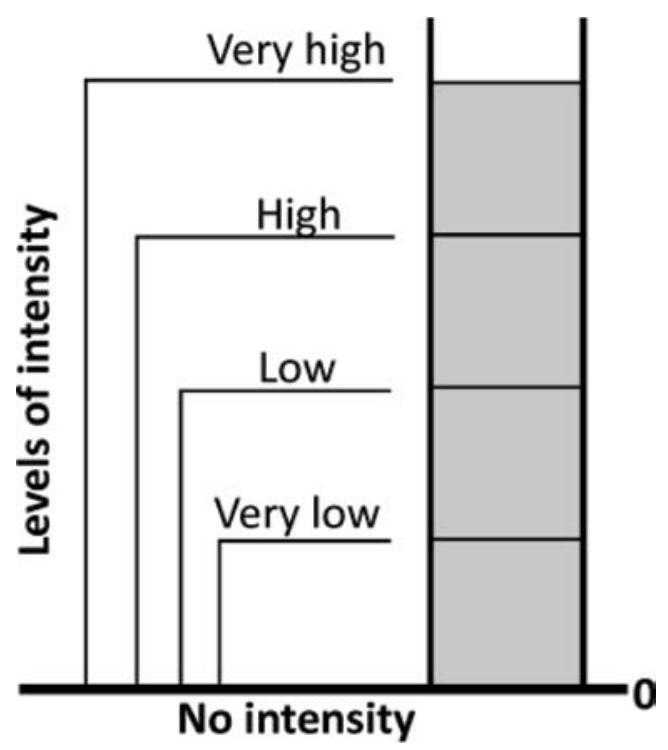

FIGURE 3 Nested character of intensity. Note that all perceptible levels of intensity include the levels that are directly below them.

about without recourse to conceptual metaphors (Raykowski 2018). They are best described in terms of levels, as depicted in Figure 3 in which a 'very high level' of intensity already includes a 'high level', which includes a 'low level', which includes a 'very low level', which in turn includes no intensity.

Another difficulty is related to the sense of privacy associated with intensity. As a private experience, the intensity represents a smaller scale (the internal state of a unit such as a neuron cell), and the extent of the intensity stands for the larger scale at which the unit (e.g., sensory cell) is replicated. Unlike the act of replicating, intensity is a static concept because it involves comparisons: to compare two sensations (e.g., of objects), they must remain in place unchanged. According to Raykowski, "This is true of nesting in general. Nesting is therefore a static mode of being - for something to nest something else, which nests something else, and so on - all nested entities have to be present at the same time" (Raykowski 2014: 136).

Another source of the conceptual difficulty involves the orthogonal relationship between intensity and extent. The two phenomena are incompatible, which has important consequences for their associations - both intensive and extensive orthogonyms must be specified for their associations to be simulated. One example of such an association is the PRODUCT schema. ${ }^{9}$ Products

9 Conflation of intensity and extent is an example of different associations of orthogonyms. It provides the prototype for the notion of accumulation, differentiation as well as the concept 


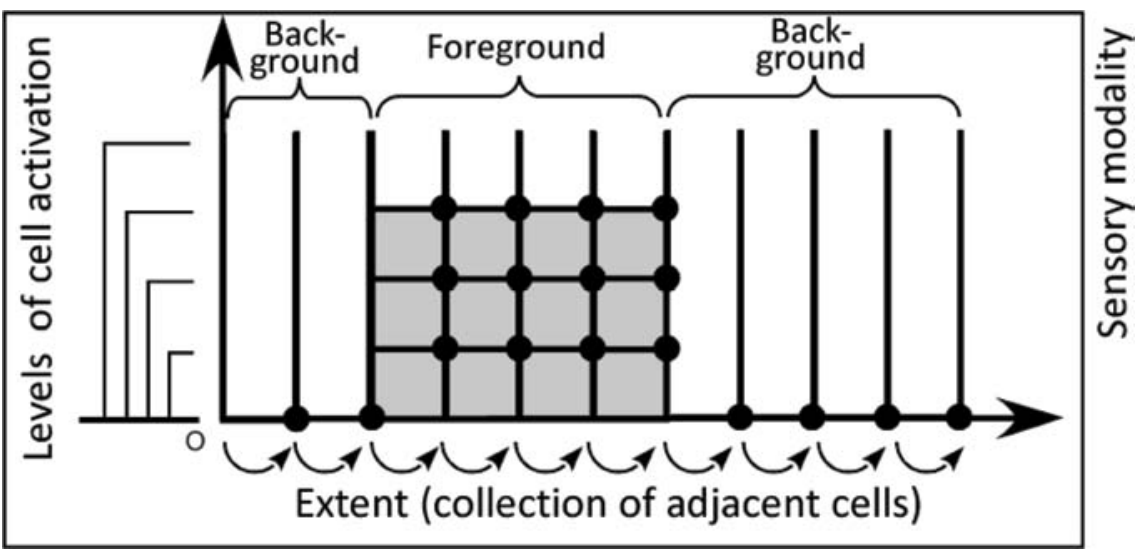

FIGURE 4 Sensory products of four activated cells from Figure 2 'dissected' along the line marked by gray. Note that the products of some intensities exist on the background of no intensity.

are 'constructed' from pairs of intensive and extensive orthogonyms by a particular way of associating them, which is depicted in Figure 4. This topic is discussed in the next section.

\section{Sensory Products and Their Relation to Orthogonyms}

The idea of products can be found in many aspects of everyday life, the economy, manufacturing, and agriculture. These ideas can be generalized as a relationship between actions and the resulting outcomes that have some value. Commercial products, for example, are objects of value produced by means of various repetitive activities. From their own experience, most readers know that the value of products can change over time and that it is possible to make products of little or no value. Note that the value assigned to a product is a nested concept in the sense that if the product costs $\$ 15$, its value already includes $\$ 10$, which includes $\$ 5$, which in turn includes $\$ 0$ value. This is typically represented as an inequality: $\$ 15>\$ 10>\$ 5>\$ 0$. The activity, on the other hand, is by nature repetitive in the sense that the act or process must be repeated each time another product is made.

of dimension, scales and various gradation systems. These topics are not discussed in this article (see Raykowski 2014: 173-174, 2018: 119-120). 
Sensory products ${ }^{10}$ share the structure outlined above; they associate intensive orthogonyms with extensive orthogonyms. The properties of sensory products can be best analyzed and described with the analogy of a water column, where products are defined as the totality of unique associations between levels of water in a container and the number of containers (Raykowski 2018). This is possible because levels represent a nested concept, and adding containers constitutes a repetitive act of some extent. The product in Figure 4, for example, represents 12 unique associations between three levels of water in four containers. Note that vessels with no water do not contribute to the product. However, they provide the background for all vessels that hold water.

Similar to levels of a liquid, cell activation is a nested concept. Whenever three levels of activation are differentiated, level 3 contains level 2 , which contains level 1 , which in turn contains level 0 , which stands for all those cells that are not activated. Note that there can be no sensation without a sensory cell in the same way that there can be no levels without a container and the column of water it forms. Because intensity and all nested concepts are expressed in terms of vertical orientation (e.g., high or low intensity / value / magnitude / amplitude) and unit repetition is expressed in terms of horizontal orientation (e.g., repeating a unit such as a container, cell, cycle, or step), these factors can be referred to as orthogonyms because their relationship is by definition perpendicular. As an example, consider six coins of two dollar values:

$$
\$ 2_{\text {value }} \cdot 6_{\text {extent }}=(()) \cdot 6=(())(())(())(())(())(())=\$ 12 \text { cognitive product }
$$

In the context of cognitive products, the concepts of value and its extent are orthogonal to each other. Observe that they are not in opposition in the way that expressions $+\$ 12$ and $-\$ 12$ are (in the sense of credit and debit). Also note that the notion of orthogonality means more than just perpendicular orientation. The terms south and east or vertical and horizontal describe perpendicular relations, but these pairs are not orthogonal. This is because products combine the smaller scale of a unit's state with the larger scale of a unit's repetition, but they also combine the notions of private and public and the notions of state and process (see Section 4.2).

By this measure, pairs such as south-east or vertical-horizontal cannot be orthogonyms, as they occupy the same scale. This also means that orthogonal concepts can only be associated and never added.

10 The reference to sensory, perceptual or cognitive products reflects the level at which those concepts are considered. 
Observe that different combinations of intensity and extent $\left(12_{\text {intensity }} \cdot 1_{\text {extent }}=\right.$ $6_{\text {intensity }} \cdot 2_{\text {extent }}=4_{\text {intensity }} \cdot 3_{\text {extent }}=3_{\text {intensity }} \cdot 4_{\text {extent }}=2_{\text {intensity }} \cdot 6_{\text {extent }}=1_{\text {intensity }} \cdot$ $12_{\text {extent }}=\$ 12$ ) result in the same cognitive product. This means that in the context of maps, products describe at least two different spatial patterns: intensive representation of the product, the extent of which is one (e.g., $\$ 12_{\text {intensity }}$. $1_{\text {extent }}$ ), and various extensive representations, the extent of which is greater than one (e.g., $\$ 1_{\text {intensity }} \cdot 12_{\text {extent }}$ or $\$ 4_{\text {intensity }} \cdot 3_{\text {extent }}$ ). ${ }^{11}$ In the example just quoted, $\$ 12$ is the product of six different yet related patterns. In the sense described in this section, sensory maps and their products represent the most basic way of thinking, which is likely reflected in all subsequent cognitive processes, including natural language. The idea of products is therefore important not only for science and mathematics but also for linguistics.

As associations of orthogonyms, cognitive products have no independent existence. Because orthogonyms are incompatible with each other, both factors must be recalled at the same time whenever their product is invoked. ${ }^{12}$ Consider orthogonyms in the context of money. The amount $\$ 532$ is a product of two orthogonyms: \$1 value and the extent of $53^{2}$. Note that the decimal number $53^{2}$ is an association of three products involving different orthogonyms: five hundreds, three tens, and two ones. In such expressions, orthogonyms are always displayed together with the manner of their own association (e.g., decimal notation). This is not always the case in natural language. Consider the phrase green leaves, beautiful summer, or cool days. It is not at all clear what kind of orthogonyms one should expect, if at all. However, before this issue is considered, the notion of embodied simulation, in which orthogonyms and their products play a critical role, needs to be discussed. This topic is addressed in the next section.

\section{Simulating Sensory Products}

The claim that orthogonyms are important for natural language cannot be made without briefly discussing the issue of embodied simulation. The question is: What exactly is activated during simulation? This question cannot

11 Intensive representations are routinely used in comparisons (e.g., of length, weight or brightness). Extensive representations depict the repetition of a unit (e.g., addition or subtraction). In addition to intensive and extensive representations, Raykowski (2018) identifies square representation, in which the intensity and its extent are 'identical'.

12 Note that the notion of products is one of a few ways in which orthogonyms can be associated. 
be answered directly because most simulation processes are automatic and unconscious both during the production and comprehension of linguistic expressions (Johnson 2015). Bergen (2012) describes this situation succinctly: "most of the simulations people construct while understanding language go completely undetected -- they're there even when people aren't aware of them." One answer to the question can be found in the analysis of nonlinguistic expressions. Many if not all concepts in science are defined in terms of intensity and extent. Their structure suggests that similar arrangement should also be present in natural language. The research on cortical maps in the brain and their role in embodied simulations provides the second answer. According to Hesslow (2012: 1), "perception can be simulated by internal activation of sensory cortex in a way that resembles its normal activation during perception of external stimuli." From the discussion in the previous sections, we know that maps in the sensory cortex code sensory activation in terms of intensity and its extent, which can be observed both in neuroscientific experiments and reports of human experiences.

The sensation of touch can be used as an example of sensory perception. It is typically experienced and tested in terms of intensity and extent of pressure. To simulate the experience, the region(s) of the sensory cortex activated during the perception of external stimuli needs to be reactivated in terms of the intensity, extent and their patterns. In the case of uniform pressure, the simulation is a product of the pressure sensation and the number of adjacent cells subjected to the pressure of identical or similar levels. The areas of varied pressure are experienced as a collection of separate products defined by their specific extents and intensities. In both cases, the brain requires a pair of orthogonal factors to simulate sensory experiences. The essential information for embodied simulations can only come from the memory of past experiences or from linguistic (or non-linguistic) expressions. The problem with natural language is that orthogonyms are often not specified or are specified in a way that is not always obvious. This issue is discussed in the next section and its subsections.

To understand natural language as a biological phenomenon, one needs to investigate processes in the brain leading to sensory experiences and analyze expressions of those sensations to identify common aspects shared by both fields of the investigation. One such link is the notion of orthogonyms. It is clearly present in most, if not all, expressions in visual art and music as 
well as in mathematics and science in general, but apparently absent from natural language. To test this, I consider a few examples of orthogonyms and their products in arts, which are more direct in their expression and perception, followed by product schemas in sciences. Orthogonyms in language are discussed last due to the complexity (hence ambiguity) of their expressions.

\subsection{Orthogonyms in Art}

Sensory experience and its expressions are associations of two orthogonyms: the intensity and extent of intensity of a sensory modality (e.g., vision, hearing, touch, or smell). I limit my discussion to the expressions of colors and sounds:

[1] Experience or expression of sound ${ }_{\text {product }}=$ $=\left(\text { pitch }_{\text {intensive factor }} \cdot \text { duration }_{\text {extensive factor }}\right)_{\text {instrument }}$

[2] Experience or expression of silence ${ }_{\text {product }}=$ $=\left(\right.$ zero pitch $_{\text {intensive factor }} \cdot$ duration extensive factor $_{\text {instrument }}$

[3] Experience or expression of color product $=$ $=\left(\right.$ intensity $_{\text {intensive factor }} \cdot$ area extensive factor $_{\text {color }}$

[4] Experience or expression of blackness ${ }_{\text {product }}=$ $=\left(\text { zero intensity }_{\text {intensive factor }} \cdot \text { area }_{\text {extensive factor }}\right)_{\text {color }}$

As mentioned previously, one cannot experience or express the intensity of a color or the pitch of a sound without sensing at least some area and some duration. At the same time, it is impossible to experience or express the extent of the color and duration of a sound without intensity and pitch. Pitch is always connected with duration [1] and intensity with an area [3]. They cannot be separated, as they are always experienced and expressed together. This includes the experience and expression of silence [2] and blank areas [4]. Because the notions of intensity and pitch are both nested, which is typically expressed in terms of vertical orientation (higher/lower value or pitch), and the notions of area and duration are usually conceptualized in terms of horizontal orientation, pairs such as the 'intensity-extent' of a color and 'pitch-duration' of a sound are orthogonyms. Other related terms that are orthogonal to the notion of area include value, brightness, lightness, luminosity and tint/shade. In the context of sound, frequency and loudness are orthogonal to duration. Note that many orthogonal concepts in art have dedicated names.

\subsection{Orthogonyms in Science and Technology}

Of all fields, orthogonyms and their products are easiest to identify in science. 
[5] Total cost product $=(\text { unit cost })_{\text {intensive factor }} \cdot($ number of units $)$ extensive factor

[6] Distance $_{\text {product }}=(\text { speed })_{\text {intensive factor }} \cdot($ time $)$ extensive factor

[7] $\quad$ Mass $_{\text {product }}=(\text { density })_{\text {intensive factor }} \cdot($ volume $)$ extensive factor

[8] Energy $_{\text {product }}=(\text { power })_{\text {intensive factor }} \cdot($ time $)$ extensive factor

In the context of their respective products, 'unit cost - number of units' [5], 'speed - time' [6], 'density - volume' [7] and 'power - time' [8] are all pairs of orthogonyms. They are either expressed with dedicated words (e.g., speed intensive factor time extensive factor distance product; density intensive factor volume extensive factor mass product) or clarified with additional descriptors (e.g., unit cost intensive factor versus total cost product). Orthogonyms in science are relatively easy to identify because their names are unique. This is not always the case in natural language expressions, which is the topic of the next section.

\subsection{Orthogonyms in Language}

Before the simulation process commences, the reader must 'determine' both the intensity of a property and its extent for at least some properties and abilities attributed to the object or subject of expression. Identifying orthogonyms in linguistic expressions is not an easy task, yet most people in most situations do this automatically. Because those processes are unconscious, they are difficult to notice, describe and discuss. Consider the apple phrase again.

\section{[9] Several red apples =}

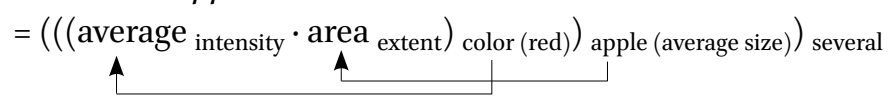

The phrase in [9] suggests the presence of two cognitive products. In the case of the first product, both factors are given indirectly, as depicted with arrows. One arrow relates intensity to red color. The human eye can distinguish a wide range of intensities, from light tints of red to dark tints of red. Whenever the intensity of the color is not specified in an expression, it must be 'assumed' by the reader to be average or typical. The notion of color, in turn, suggests the surface area as an extent of the intensity, which is defined by the noun (apple). In the absence of actual experience, the surface is visualized as that of an average or typical apple.

The second product in the phrase [9] is created by repeating the intensive representation of the first product several times. ${ }^{13}$ Because there are two products, there must be two sets of orthogonyms. The factors in the phrase 'several

13 A product of more than two factors follows the same pattern: $\$ 2_{\text {intensity }} \cdot 5$ extent $\cdot 3$ extent $=$ $\left(\$ 2_{\text {intensity }} \times 5_{\text {extent }}\right) \cdot 3$ extent $=\$ 10_{\text {extensive representation }} \cdot 3_{\text {extent }}=\$ 10_{\text {intensive representation }} \cdot 3_{\text {extent }}=\$ 30$. 
apples' are easy to identify in the context of color: the 'apple' (in this case, the color of its surface) is an intensive factor, and 'several' stands for the extent of repetition. The relationship between those factors can be described as that of an intensive unit and its multitude, which are orthogonal to each other. The orthogonyms in the phrase 'red apple' are less obvious. They include the average intensity of the property (in this case, color) and its area (the surface of an average-size apple), which can be described in general terms as intensity and extent.

\subsection{More Examples of Orthogonyms in Language}

The issue of orthogonyms is non-trivial due to the extensive use of those structures in natural language. As an example, consider an excerpt from Mark Twain's novel "The Prince and the Pauper":

[10] Before him, at a little distance, reclined a very large and very fat man, with a wide, pulpy face, and a stern expression. (Twain 1882: Chapter 5)

The excerpt in [10] contains several phrases acting as prompts for embodied simulations: [10a] 'at a little distance', [10b]'a very large man', [10c] 'a very fat man', [10d] 'a wide face', [10e] 'a pulpy face', and [1of] 'a stern expression'. To experience a cognitive product by means of simulation, both orthogonal factors have to be activated.

As an example, consider the opening phrase [10a]. Most readers would concur that humans understand distance in terms of walking, which could be defined as an act of repeating a step of some length. The phrase could therefore be translated into such walking-based expressions as a few steps away or a small number of steps away, which are much easier to analyze. Steps come in different sizes. Their lengths are nested in the sense long step $>$ medium step $>$ short step $>$ no step. Any one of those steps could be an intensive factor of the product (intensive orthogonym), but which step is adopted is usually defined by the phrase under consideration. If the length of the step is not specified, readers must assume either an average or the most comfortable step. Note that the word 'distance' used in the phrase refers to the product arrived at by repeating the step a number of times, as suggested by the words 'a little. The phrase 'a little' could be interpreted as 'small' as in 'small distance' (intensive representation of the product) or as 'small number' as in a 'small number of steps' (extensive representation). Note that the phrase 'a little' can be contrasted with 'a

The extensive products must be converted to the intensive representation before they can be repeated. 
lot', which clearly suggests repetition of a unit. The orthogonyms in this phrase are two concepts: 'medium step - a little' or a more generally nested unit and its repetition.

The phrase $[10 \mathrm{~b}]$ ' $a$ very large man' follows a similar pattern. The article 'a' suggests the extent of 'one', which is characteristic of intensive representations of products. This in turn suggests a comparison:

(very large $>$ large $>$ medium $>$ small $>$ very small $>0$ ) ${ }_{\text {man }}$, constituting the nested aspect of the property 'large'. The expression describes the subject as substantially larger than the typical man.

[10b] $\quad\left(\left(\left(\right.\right.\right.$ very $\left._{\text {intensity }} \cdot \mathbf{1}_{\text {extent }}\right)$ large = heavy/tall/yoluminous $)$ 'a' (article $)$ man (noun)

Note that the adjective 'large' can refer to volume, weight, height and width. The phrase does not specify any particular aspect of the property and therefore it can be understood as a combination of those attributes. The orthogonyms in this phrase are a pair of concepts: a nested factor of the property (very) large $_{\text {and }}$ and the extent of its repetition (one) or, more generally, magnitude and multitude.

The phrases [10d] and [10e] are slightly different. The intensive factor of their products is not stated; hence, they must be assumed as typical (medium or average). The remaining phrases follow identical patterns and hence are not analyzed in detail. For convenience, the orthogonyms of all phrases in this section are assembled in the table below, which makes patterns that all orthogonyms follow easier to notice and analyze.

\begin{tabular}{|c|c|c|c|c|c|}
\hline Reference & Phrase & $\begin{array}{l}\text { Intensive } \\
\text { orthogonym }\end{array}$ & $\begin{array}{l}\text { Extensive } \\
\text { orthogonym }\end{array}$ & Property & Entity \\
\hline$[10 \mathrm{a}]$ & $\begin{array}{l}\text { At a little } \\
\text { distance }\end{array}$ & Medium step & A little & Length & Distance \\
\hline$[10 \mathrm{ob}]$ & $\begin{array}{l}\text { A very large } \\
\text { man }\end{array}$ & Very & One & Large-ness & Man \\
\hline$[10 c]$ & A very fat man & Very & One & Fat-ness & Man \\
\hline [1od] & A wide face & Average & One & Wide-ness & Face \\
\hline$[10 \mathrm{e}]$ & A pulpy face & Average & One & Pulpy-ness & Face \\
\hline$[10 f]$ & $\begin{array}{l}\text { A stern } \\
\text { expression }\end{array}$ & Average & One & Stern-ness & Face \\
\hline
\end{tabular}

The expressions in the table above represent visual patterns experienced in terms of length, area or volume. Some phrases give rise to more complex 
simulations than others. For example, a pulpy face is not just round but also soft and squashy, and a stern face can be seen as unkind, unyielding, and steadfast. Which of those sub-features are simulated depends not only on the prior experience but also on a cognitive predisposition of the person (Bergen 2012). Some individuals are 'innately more visual' and therefore, their simulations are highly 'realistic'. Other individuals are more tactile and kinesthetically orientated and hence, their visual simulations are schematic. Therefore, even if they read the same text, different individuals tend to invoke different aspects of the experience. Despite the difference, those simulations are likely to have the orthogonal structure outlined in this paper.

\section{$8 \quad$ Conclusion}

Thinking in terms of orthogonyms and their products may seem to be a pointless complication of familiar linguistic principles. From the point of view of cognitive semantics, however, the conceptual analysis proposed in this paper makes sense because "semantics is specifically concerned with the conceptual organisation of language" (Talmy 2003: 4). Evans and Green (2006: 157) reinforce this interpretation further:

A fundamental concern for cognitive semanticists is the nature of the relationship between conceptual structure and the external world of sensory experience. In other words, cognitive semanticists set out to explore the nature of human interaction with and awareness of the external world and to build a theory of conceptual structure that is consonant with the ways in which we experience the world.

The hypothesis of orthogonyms is an example of such a theory.

Many patterns in cognitive linguistics (e.g., image schemas, metaphors) are relatively easy to notice, comprehend and discuss. Products and orthogonyms, on the other hand, are difficult to identify for a variety of reasons, some of which include the underspecified nature of language, the use of the same word for different concepts, the reliance on context for the meaning of words, and the unconscious and automated nature of the processes involving orthogonyms and their products. It must be appreciated that cognitive products and their factors in natural language are not grammatical but conceptual structures. They do not follow norms and standards of phonetics, morphology, syntax, semantics, and pragmatics - rather, they follow the logic of sensory experiences accumulated over the span of one's life. It is therefore unreasonable to expect 
familiar content and composition or hope for dedicated names for products and each of their two factors. More often than not, orthogonyms in language can be described only in the most general terms, such as 'intensity-extent', 'magnitude-multitude', 'value-repetition', etc.

As noted before, orthogonyms are much easier to identify in art, science and technology than in natural language. Therefore, those forms of expression can be used in future research as a model for untangling the issue of orthogonyms and their products in linguistics. This follows directly from the generalization and cognitive commitments, which insist that the organization of language should reflect general cognitive principles. In other words, if orthogonyms and cognitive products can be found in fine art, science and technology, they must also be present in natural language.

\section{References}

Bacchetta, Marc, Cosimo Beverelli, Oliver Cadot, Marco Fugazza, Jean-Marie Grether, Matthias Helble, Alessandro Nicita, and Roberta Piermartini. 2012. A Practical Guide to Trade Policy Analysis. Geneva: World Trade Organization and United Nation.

Barsalou, Lawrence. 1999. Perceptual symbol systems. Behavioral and Brain Sciences 22: 577-6o9.

Bednar, James and Stuart Wilson. 2015. Cortical maps. The Neuroscientist 22(6): 604-617.

Bergen, Benjamin. 2012. The New Science of Meaning. Huffingtonpost. Available as download http://www.huffingtonpost.com/benjamin-k-bergen/the-new-science-of -meaning_b_227309o.html.

Caldwell, John. 2009. Action potential initiation and conduction in Axons. Encyclopedia of Neuroscience, 23-29.

Evans, Vyvyan and Melanie Green. 2006. Cognitive Linguistics: An Introduction. London: Lawrence Erlbaum Associates.

Fairlie-Clarke, Anthony. 1999. Force as a flow variable. Proceedings of the Institution of Mechanical Engineers 213(1): 77-81.

Gardner, Esther and Kenneth Johnson. 2012. Sensory coding. In Eric R. Kandel, James H. Schewartz (eds.) Principles of Neural Science, 449-474. McGraw Hill Companies. Grodd, Wolfgang, Ernst Hülsmann, Martin Lotze, Dirk Wildgruber, and Michael Erb. 2001. Sensorimotor mapping of the human cerebellum: fMRI evidence of somatotopic organization. Human Brain Mapping 13 (2): 55-73.

Harnad, Stevan. 1987. Categorical Perception: The Groundwork of Cognition. New York: Cambridge University Press.

Hesslow, Germund. 2012. The current status of the simulation theory of cognition. Brain Research 1428: 71-79. 
Hezemans P. M. A. L., and van Geffen L. C. M. M. 1991. Analogy theory for a system approach to physical and technical systems. In Fishwick P. A. and Luker P. A. (eds.) Qualitative Simulation Modeling and Analysis, 170-216. New York: Springer.

Johnson, Mark. 1987. The Body in the Mind: The Bodily Basis of Meaning, Imagination, and Reason. Chicago: University of Chicago Press.

Johnson, Mark. 2015. Embodied understanding. Frontiers in Psychology 6: 1-8.

Lakoff, George and Mark Johnson. 2003. Metaphors We Live By. London: The University of Chicago Press.

Langacker, Ronald Wayne. 1987. Foundations of Cognitive Grammar, Vol.1: Theoretical Prerequisites. Stanford: Stanford University Press.

Nunes, Terezinha and Peter Bryant. 20og. Paper 2: Understanding whole numbers. Key Understandings in Mathematics Learning, 1-38. London: Nuffield Foundation.

Penfield, Wilder, and Edwin Boldrey. 1937. Somatic Motor and Sensory Representation in the Cerebral Cortex of Man as Studied by Electrical Stimulation. Brain 6o(4): 389-443.

Ramón y Cajal, Santiago. 1899. Comparative Study of the Sensory Areas of the Human Cortex. p. 85. Archived from the original on 10 September 2009.

Raykowski, Wes. 2014. Conceptual Understructure of Human Experience: Volume 1 (Thesis). CreateSpace, North Charleston, South Carolina.

Raykowski, Wes. 2018. Layers and levels: What a column of water tells us about human cognition. Cognitive Semantics 4: 104-134.

Smith, Christopher. 200o. Biology of Sensory Systems. John Wiley \& Sons.

Smith, Douglas. 2009. Stretch growth of integrated axon tracts: Extremes and exploitations. Progress in Neurobiology 89(3): 231-239.

Talmy, Leonard. 2000. Toward a Cognitive Semantics, Volume 1: Concept Structuring Systems. Cambridge, MA: MIT Press.

Twain, Mark. 1882. The Prince and the Pauper. Available as download https:// en.wikisource.org/wiki/The_Prince_and_the_Pauper/Chapter_5.

Tyler, Andrea and Vyvyan Evans. 2003. The Semantics of English Prepositions: Spatial Scenes, Embodied Meaning and Cognition. Cambridge: Cambridge University Press.

Wang, Irving and Thomas Clandinin. 2016. The influence of wiring economy on nervous system evolution. Current Biology 26(20): R1101-R1108. 